\title{
Performance test on PELICAN - a multi-purpose time of flight cold neutron spectrometer
}

\author{
Dehong Yu', Richard. A. Mole and Gordon J. Kearley \\ Bragg Institute, Australian Nuclear Science and Technology Organisation, New Illawarra Road, Lucas Heights, 2234, \\ Australia
}

\begin{abstract}
Pelican, a direct geometry multi-purpose cold neutron spectrometer has recently been commissioned at the Bragg Institute, ANSTO. The energy resolution and flux at the sample position as a function of neutron wavelength has been evaluated and time focusing at selected energy transfers has also been demonstrated. Several test experiments of quasi-elastic and inelastic neutron scatterings have been performed and these have indicated the realisation of the design specifications and performance of the instrument.
\end{abstract}

\section{Introduction}

The direct-geometry, time-of-flight neutron spectrometer, Pelican, has been installed and commissioned at the OPAL research reactor, at the Australian Nuclear Science and Technology Organization (ANSTO). The Pelican instrument was designed to meet the diverse requirements of the Australian scientific community from physics, chemistry and materials science, to biology. A wide range of research fields will be covered; these include crystal-field excitations, phonon densities of states, magnetic excitations from various multifunctional materials including high Tc superconductors, novel magnetic, thermo-electric, ferroelectric and piezoelectric materials; molecular dynamics in hydrogen-bonded and hydrogen storage materials, catalytic materials, cements, soils and rocks; and water dynamics in proteins and ion diffusion in membranes. A polarisation analysis option is also included in the design of the instrument [1].

Pelican is located on the CG1 cold neutron guide. The neutron wavelength covers from $2.4 \AA$ to $6.3 \AA$ (14.2 meV to $2.1 \mathrm{meV}$ ), delivered by a specially designed and fabricated triple monochromator system, consisting of three banks of highly oriented pyrolytic graphite (HOPG) crystals having 0.5 degree of mosacity [2]. Combined with high speed Fermi choppers, energy resolution in the range $0.06 \mathrm{meV}$ to $1.5 \mathrm{meV}$ can be achieved at the elastic line, while the energy resolution at a desired energy transfer can be optimised through time focusing achieved by adjusting the speed and the sense of rotation of the Fermi chopper to allow the slow neutrons pass the chopper before the fast ones so that they arrive at the detector at the same time [1]. In this paper, the performance of the instrument will be demonstrated with the results of measured energy resolution and flux at the sample position as a function of neutron wavelength and the evaluation on several real systems with quasi-elastic and inelastic

\footnotetext{
${ }^{a}$ Corresponding author: dyu@ansto.gov . au
}

neutron scatterings. For the general design specifications of the Pelican instrument and descriptions of major components such as monochromator, polarizer, master and slave Fermi choppers, vacuum chambers and detector system, please refer to reference [1]. The commission and performance test of the polarisation analysis system will be presented later elsewhere.

\section{Energy resolutions and flux}

\subsection{Energy resolutions}

The energy resolution as a function of neutron wavelength has been measured on a cylindrical vanadium sample at room temperature. The diameter and height of the vanadium sample is $20 \mathrm{~mm}$ and $60 \mathrm{~mm}$ respectively, with wall thickness of $0.5 \mathrm{~mm}$. Figure 1 shows the full width at half maximum (FWHM) of the elastic line fitted with a Pseudo-Voigt function which was found to fit the data slightly better than a Gaussian function, though the same values of FWHM are obtained from both functions. The solid line is as a guide for the eye only. These values all have optimised time focussing at the elastic line with frequencies in the range 65 to $120 \mathrm{~Hz}$ as reported in reference [1] A Be-filter was used for neutron wavelength above $4.0 \AA$ and a graphite filter was used for $3 \AA$ neutrons for both resolution and flux evaluations.

\subsection{Flux at sample position}

Figure 2 shows the measured monochromatic, pulsed neutron flux $\left(\mathrm{n} / \mathrm{cm}^{2} / \mathrm{s}\right)$ at the sample position for various incident wavelengths and a wavelength distribution of $\Delta \lambda / \lambda$ of about $2 \%$, with the solid line as a guide for eye. The measurements were taken with a U-235 beam monitor calibrated against the measurement with gold foil method for $4.7 \AA$ neutrons at the sample position. The gold foil measurement was taken for 12 hours, while the measurements with beam monitor were carried out over 0.5 hour. Thus the neutron flux reported here is 


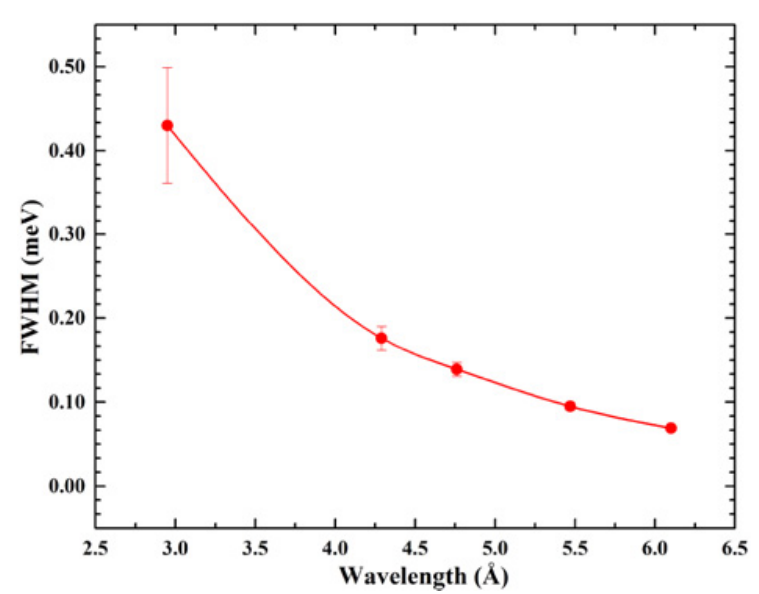

Figure 1. Energy resolutions versus neutron wavelength, measured with a standard vanadium sample corresponding to time focusing at elastic line.

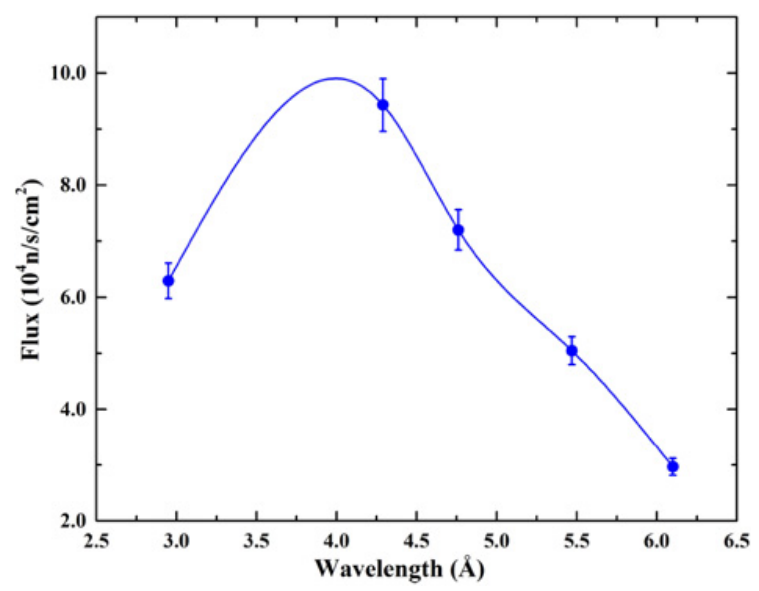

Figure 2. Pulsed neutron flux versus wavelength at sample position with chopper frequency $100 \mathrm{~Hz}$.

corresponding to the time averaged values over many pulses. Corrections of wavelength dependence of the gold foil and the beam monitor have been taken and the overall uncertainty of the flux is about $5 \%$. It is noted that maximum flux of $9.4 \times 10^{4} \mathrm{n} / \mathrm{cm}^{2} / \mathrm{s}$ has been measured at $4.3 \AA$. This is comparable to that from the similar instruments like FOCUS instrument at PSI [3] and IN6 instrument at ILL [4]. For comparison purpose, the same Fermi chopper frequency of $100 \mathrm{~Hz}$ was used for these measurements. This frequency corresponds to the focusing condition for $5.5 \AA$ neutrons. Please note that the neutron flux is not changed by the time focusing condition which only alternates the energy resolution by letting neutrons with slightly different speeds going through the chopper at slightly different time [1].

\subsection{Time focusing}

The advantage of the Fermi chopper arrangement is its capability for time focusing in combination with the triple monochromators. By adjusting the frequency and the sense of rotation of the Fermi chopper, the rotating slit package can scan the monochromators in such a way to let the slow neutrons pass before the fast ones so that they arrive

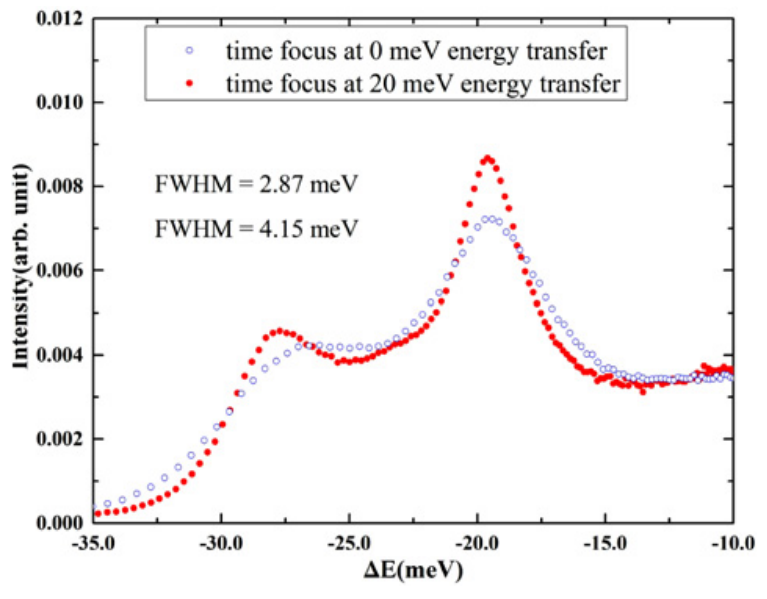

Figure 3. Demonstration of time focusing capability with the vanadium phonon density of state; empty circle corresponds to time focusing at elastic line; filled circle is with time focusing at $20 \mathrm{meV}$ energy transfer.

at the detector at same time $[1,5]$. The time focusing performance has been demonstrated with the vanadium sample as shown in Fig. 3. At neutron wavelength of $4.3 \AA$, two measurements were performed with different chopper frequencies corresponding to time focusing at elastic line $(75 \mathrm{~Hz})$ and at energy transfer of $20 \mathrm{meV}(200 \mathrm{~Hz})$ around the position of the vanadium phonon density of states. By fitting the peak around $20 \mathrm{meV}$ with a Pseudo-Voigt function, the FWHM of $4.15 \pm 0.05 \mathrm{meV}$ and $2.87 \pm$ $0.05 \mathrm{meV}$ were obtained with the chopper frequencies of $75 \mathrm{~Hz}$ and $200 \mathrm{~Hz}$, respectively. About 30\% improvement in energy resolution around $20 \mathrm{meV}$ energy transfer was achieved with $200 \mathrm{~Hz}$ chopper frequency as compared with the case of $75 \mathrm{~Hz}$ chopper frequency. However the elastic line resolution only increases by approximately $5 \%$ when the time focussing condition was moved to $20 \mathrm{meV}$, in the above example the elastic resolution changed from $0.20 \mathrm{meV}$ to $0.21 \mathrm{meV}$ for 75 and $200 \mathrm{~Hz}$ respectively without noticeable change in line shape of the elastic peak.

In the above measurements the stated chopper frequency is that of the master chopper. For the $75 \mathrm{~Hz}$ data, both the master and slave chopper were $75 \mathrm{~Hz}$ (one-to-one chopper ratio). For the $200 \mathrm{~Hz}$ data a two-to-one chopper ratio was used, with the slave chopper running at $100 \mathrm{~Hz}$. This was done to avoid frame overlap.

\subsection{HOPG higher order reflection}

Recent work has also focussed on extending the possible energy transfer range that can be measured using Pelican. Typically the wavelength range is restricted by the accessible monochromator two-theta range; however shorter wavelengths are available in the source spectrum and these can be accessed by utilising higher order reflection from the monochromator (ie using the PG 004 reflection to obtain $\lambda / 2$ ). The separation between the master and slave Fermi choppers on Pelican is $750 \mathrm{~mm}$. By phasing the choppers one can filter $\lambda$ from the transmitted beam. Using no Be-filter, this would still result in a beam contaminated with $\lambda / 3$ and $\lambda / 4$ etc. However pelican is also equipped with a tuneable graphite filter. This can 




Figure 4. Data collected with $\lambda=4.3 \AA$ on vanadium sample, with varying filter and chopper configurations: blue line, no filter in beam, choppers phased to allow maximum transmission of $\lambda$. Black line, No filter in beam, choppers phased to minimise the transmission of $\lambda$. Red line, Graphite filter in beam, choppers phased to minimise the transmission of $\lambda$.

be optimised to filter $\lambda / 3$ and $\lambda / 4$ and still transmit a significant fraction of $\lambda / 2$. Such a configuration has recently been tested and shown that a wavelength of $2.15 \AA$ can be successfully used on Pelican as indicated in Fig. 4. The data were taken with a vanadium sample, using a $\lambda$ of $4.30 \AA$. The choppers were run at $75 \mathrm{~Hz}$ (one-toone ratio), corresponding to the elastic line time focusing condition. At this condition, it is not expected to have significant broadening on the $2.15 \AA$ elastic peak, as the time focusing frequency does not change rapidly with neutron wavelength in the short wavelength range [1]. The blue line shows the data integrated over all angles with no filters in the beam and the choppers phased to allow $\lambda, \lambda / 2, \lambda / 3$ and $\lambda / 4$. In this configuration there are several other spurions that cannot be easily identified. The black line shows the same instrument configuration, but with the choppers phased so that no $\lambda$ neutrons are transmitted. Finally the red line is the same chopper phasing, but with the graphite filter in the beam. Now the main peak transmitted is $\lambda / 2$, with only a small contamination of $\lambda / 3$. There are no other clear spurions in the beam with this configuration.

\section{Test experiments}

So far several scientific experiments of quasi-elastic (QENS) and inelastic neutron scatterings have been performed. Here we present some results from two experiments of water dynamics in amino acid solution and crystal field excitation in $\mathrm{PrFeO}_{3}$ powder sample.

\subsection{QENS on proline solution}

In order to see the contribution of proline to water dynamics in proline solution, we measured and compared the broadening of the elastic peak from both $\mathrm{H}_{2} \mathrm{O}+$ proline and $\mathrm{D}_{2} \mathrm{O}+$ proline solutions [6], as shown in Fig. 5. Both samples contain $9.1 \%$ mole fraction of proline. The measurements were performed with $6.2 \AA$ and $0.06 \mathrm{meV}$

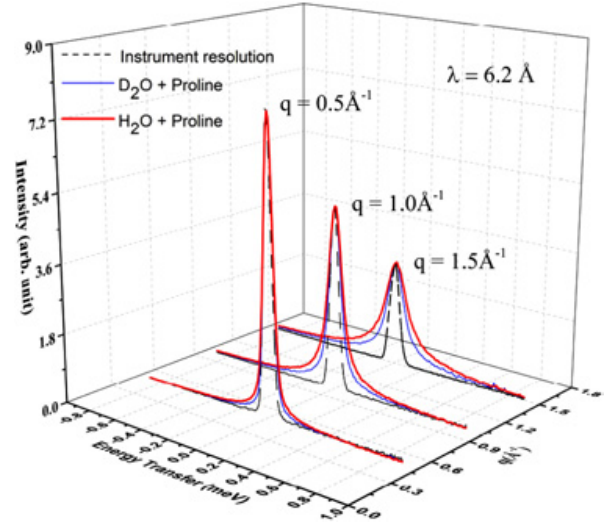

Figure 5. Experimental QENS spectra of 9.1 molar\% proline in water. The signals from the vanadium (dashed), $\mathrm{C}_{5} \mathrm{H}_{6} \mathrm{D}_{3} \mathrm{NO}_{2}$ (thin line) in $\mathrm{D}_{2} \mathrm{O}$ and $\mathrm{C}_{5} \mathrm{H}_{9} \mathrm{NO}_{2}$ (thick line) in $\mathrm{H}_{2} \mathrm{O}$ have been normalised to a common maximum to aid comparison.

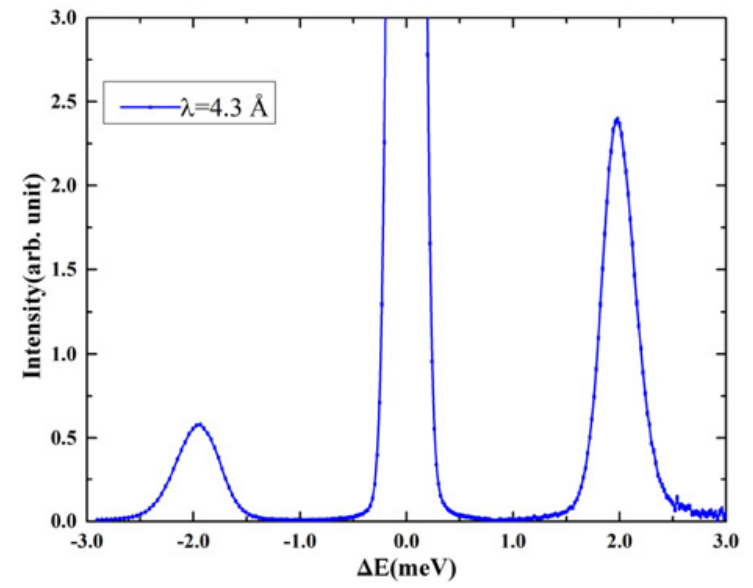

Figure 6. Crystal electric field excitation of $\mathrm{PrFeO}_{3}$, with incident neutron wavelength of $4.3 \AA$ at sample temperature of $20 \mathrm{~K}$.

energy resolution with $100 \mathrm{~Hz}$ chopper frequency, not corresponding to the elastic time focusing condition. An annular sample container was used providing a sample thickness of $0.4 \mathrm{~mm}$. Normalization to vanadium run and background subtraction of an empty sample can were performed with data reduction software LAMP [7].

\subsection{Crystal electric field excitation of $\mathrm{PrFeO}_{3}$}

Crystal electric field excitations on $8 \mathrm{~g}$ of $\mathrm{PrFeO}_{3}$ powder sample have been performed with a neutron wavelength of $4.3 \AA$ at low temperatures. The transition to the first excited state has been observed at $2 \mathrm{meV}$ energy transfer, as shown in Fig. 6 at $20 \mathrm{~K}$ temperature. The spectrum is the integration over 180 detectors covering angular range from 12 degree to 125 degree, after vanadium normalization and empty can subtraction. The measurement agrees very well with previous observation [8] and recent measurement on Hyspec instrument at SNS, USA [9]. This has provided a benchmark test of the Pelican instrument and the associated LAMP data reduction software [7]. 


\section{Conclusions}

The Pelican instrument has reached the stage for normal operation and is planned to enter user program in the second half of 2014. The polarization analysis option will be available at a later stage.

The authors would like to thank the mechanical, electrical and data acquisition team members at the Bragg Institute for their great help in commissioning the instrument. DHY would like to thank Prof. SX Cao for having provided the $\mathrm{PrFeO}_{3}$ sample.

\section{References}

[1] Dehong Yu, Richard Mole, Terry Noakes, Shane Kennedy and Robert Robinson, J. Phys. Soc. Jpn. 82 (2013) SA027

[2] A. K. Freund and D. H. Yu: Nucl. Instr. and Meth., A 634 (2011) S75
[3] S. Janssen, F. Altorfer, L. Holitzner and R. Hempelmann, Physica B 276-278 (2000) 89

[4] http://www.ill.eu/instruments-support/ins truments-groups/instruments/in6/descript ion/

[5] H. Mutka: Nucl. Inst. and Meth. A 338 (1994) 144

[6] D. H. Yu, M. Hennig, R. A. Mole, J. C. Li, C. Wheeler, T. Straessle and G. J. Kearley, Phys. Chem. Chem. Phys. 15 (2013) 20555

[7] LAMP, the Large Array Manipulation Program. http://www.ill.eu/data_treat/lamp/the -lamp-book/

[8] K. Feldmann, K. Hennig, L. Kaun, B. Lippold, M. M. Lukina, S. Matthies, W. Matz and E. Warming, phys. Stat. Sol. (b) 72 (1975) 817

[9] http://neutrons.phy.bnl.gov/CNS/hyspec/ index.htm 